\title{
Ribociclib and Abemaciclib:
}

\section{$\mathrm{CDK}_{4} / 6$ Inhibitors for the Treatment} of Hormone Receptor-Positive

\section{Metastatic Breast Cancer}

\author{
KRISTINA F. BYERS, PharmD, BCOP
}

From Emory University Winship Cancer Institute, Atlanta, Georgia

Author's disclosures of conflicts of interest are found at the end of this article.

Correspondence to: Kristina F. Byers, PharmD, BCOP, 1365 Clifton Road NE, Atlanta, GA 30322 E-mail: kristina.byers@emoryhealthcare.org

https://doi.org/10.6004/jadpro.2021.12.1.8

(c) 2021 Harborside ${ }^{T M}$

\section{Abstract}

The treatment landscape of hormone receptor-positive $(\mathrm{HR}+)$, human epidermal growth factor receptor 2-negative (HER2-) metastatic breast cancer has been modernized by the identification of cyclindependent kinase 4 and $6(\mathrm{CDK} 4 / 6)$ inhibitors. Because the majority of $\mathrm{HR}+$ breast cancers will develop resistance to endocrine therapies (tamoxifen and aromatase inhibitors), newer treatment options are necessary to restore endocrine sensitivity and prolong survival. Ribociclib and abemaciclib are two of three CDK4/6 inhibitors currently approved for first- and second-line treatment of HR+/HER2- metastatic breast cancer. Data from large, phase III clinical trials have demonstrated an improvement in both progression-free and overall survival with the addition of ribociclib or abemaciclib to endocrine-based therapy, establishing a new frontline standard of care. Treatment with ribociclib and abemaciclib provide a convenient oral treatment option that is both efficacious and well tolerated.

reast cancer is the most commonly diagnosed cancer in the United States, accounting for $30 \%$ of all new cancer diagnoses annually. It is estimated that 279,100 people were diagnosed with breast cancer in 2020. Although the development of newer therapies and better screening methods has increased breast cancer survival rates, metastatic disease is still the second most common cause of cancer-related death in women (Siegel et al., 2020). Approximately $75 \%$ of breast cancers are considered hormone receptor-positive ( $\mathrm{HR}+)$ and express estrogen and/or progesterone receptors (Anderson, Chatterjee, Ershler, \& Brawley, 2002), with endocrine therapy serving as the mainstay of systemic treatment (Ribnikar, Volovat, \& Cardoso, 2019). 
Despite the widespread use of endocrine therapy, a proportion of patients will develop endocrine resistance, leading to treatment failure and progressive disease. In the past decade, research has focused on the development of novel drug targets that aim to restore or extend endocrine sensitivity (D'Souza, Spicer, \& Lu, 2018). The addition of the cyclin-dependent kinase 4 and 6 (CDK4/6) inhibitors palbociclib (Ibrance), ribociclib (Kisqali), and abemaciclib (Verzenio) to standard endocrine therapy has significantly improved progression-free survival (PFS) as initial and second-line therapy in patients with $\mathrm{HR}+$, human epidermal growth factor receptor 2-negative (HER2-) metastatic breast cancer (D'Souza et al., 2018).

Palbociclib was the first CDK4/6 inhibitor to receive U.S. Food \& Drug Administration (FDA) approval in February 2015; however, this article will focus on the newer CDK4/6 inhibitors, ribociclib and abemaciclib, which gained FDA approval in March 2017 and February 2018, respectively. The purpose of this article is to provide the advanced practitioner with the tools necessary to manage metastatic HR+, HER2- breast cancer patients initiating therapy with ribociclib or abemaciclib. The contents of this article will focus on the mechanism of action, efficacy and safety data, dosing, monitoring, and practical implications of these agents.

\section{PHARMACOLOGY AND MECHANISM OF ACTION}

The cell cycle is regulated by several proteins, including the cyclin-dependent kinase-retinoblastoma $(\mathrm{Rb})$ signaling pathway. Specifically, cyclin D binds to CDK4/6, which results in phosphorylation of $\mathrm{Rb}$, leaving the tumor suppressor gene inactive. Once inactivated, $\mathrm{Rb}$ releases the transcription factor $\mathrm{E} 2 \mathrm{~F}$, which promotes progression from the $\mathrm{G} 1$ to $\mathrm{S}$ phase of the cell cycle, allowing for DNA replication and tumor progression. Furthermore, there is a close link between cyclin D (CCND1) and estrogen receptor-mediated transcription. Overexpression of the CCND1 oncogene, which occurs in as many as $50 \%$ of breast cancers, leads to cell cycle dysregulation and cancer cell survival, and is thought to be a mechanism of endocrine resistance (Ribnikar et al., 2019).
Ribociclib is an orally bioavailable, selective CDK4/6 inhibitor that has demonstrated efficacy in HR+, HER2 - metastatic breast cancer when used in combination with a nonsteroidal aromatase inhibitor (AI) or fulvestrant. Ribociclib is extensively metabolized via hepatic CYP3A4 enzymes to the major circulating metabolites M13, M4, and M1; however, its clinical activity is primarily attributed to the parent drug, which accounts for $44 \%$ of the circulating drug moiety. The mean terminal half-life of ribociclib is 30 to 55 hours, allowing for once daily dosing. It is primarily eliminated in the feces (69\%); only a fourth of ribociclib excretion occurs via renal elimination (Novartis Pharmaceuticals Corporation, 2020).

Abemaciclib is another oral selective CDK4/6 inhibitor that has demonstrated clinical activity alone and in combination with endocrine therapy. Abemaciclib also undergoes extensive hepatic metabolism via CYP3A4 to active metabolites M2 (primary), M20, and M18. Both abemaciclib and its active metabolites (M2 and M20) can be detected at similar concentrations in the cerebral spinal fluid and plasma (unbound). Due to a shorter mean terminal half-life compared with that of ribociclib (18.3 hours), abemaciclib requires twice daily dosing to maintain steady-state concentrations (Eli Lilly and Company, 2020). Structural differences between abemaciclib and the other CDK4/6 inhibitors account for a higher affinity for CDK4 compared with CDK6 (Spring, Zangardi, Moy, \& Bardia, 2017).

\section{CLINICAL TRIALS \\ Ribociclib}

MONALEESA-2 was a phase III, randomized, placebo-controlled trial that evaluated the benefit of adding ribociclib (600 mg daily on a 3 weeks on, 1 week off schedule) to letrozole ( $2.5 \mathrm{mg}$ daily) as frontline therapy in postmenopausal women with HR+/HER2 - metastatic breast cancer. The primary endpoint of median duration of PFS was significantly longer in the ribociclib/letrozole group ( $n=334)$ compared with the letrozole/placebo group ( $n=334$; not reached vs. 14.7 months; 95\% confidence interval $[\mathrm{CI}]=13.0-16.5)$, confirming the superiority of ribociclib/letrozole. Progression-free survival rates at 12 and 18 months were 
higher in the ribociclib/letrozole group $(72.8 \%$ and $63 \%$, respectively) compared with the letrozole/placebo group (60.9\% and $42.2 \%$, respectively). At the time of the interim analysis, overall survival (OS) data were not yet mature (Hortobagyi et al., 2016).

MONALEESA-7 was a phase III, randomized, placebo-controlled trial that aimed to evaluate the benefit of adding ribociclib to endocrine therapy and goserelin in treatment-naive premenopausal women. Prior to MONALEESA-7, treatment recommendations for premenopausal women relied on data extrapolated from studies in postmenopausal women. Patients $(n=672)$ were randomized in a 1:1 fashion to receive ribociclib $(600 \mathrm{mg}$ daily on a 3 weeks on, 1 week off schedule) plus endocrine therapy vs. endocrine therapy alone (placebo group). Selection of endocrine therapy was left to the discretion of the investigator and included tamoxifen (20 mg daily) or an AI (anastrozole $1 \mathrm{mg}$ daily or letrozole $2.5 \mathrm{mg}$ daily). All patients received ovarian suppression with goserelin (3.6 mg administered subcutaneously on day 1 of a 28-day cycle).

The primary endpoint of median PFS was 23.8 months in the ribociclib group vs. 13 months in the placebo group (hazard ratio [HR], 0.55; 95\% CI = 0.44-0.69). Within the ribociclib group, median PFS was numerically higher in patients receiving an AI compared with those receiving tamoxifen (27.5 months and 22.1 months, respectively; Tripathy et al., 2018). At the time of the initial publication, OS data had not matured; however, a prespecified interim analysis of OS was planned after 192 deaths were reported. At 42 months followup, the median OS rate was $70.2 \%$ in the ribociclib group compared with $46 \%$ in the placebo group (HR, 0.7; 95\% CI = 0.50-0.98). The OS benefit seen in the overall population was maintained in the prespecified subgroup of patients receiving an AI; however, it was not maintained in the tamoxifen group (Im et al., 2019).

Ribociclib was also evaluated in combination with fulvestrant in the phase III, randomized, placebo-controlled, MONALEESA-3 trial in postmenopausal women who were either treatment naive or had received one prior line of endocrine therapy for advanced disease. Of the 726 patients enrolled, 484 were randomized to ribociclib (600 mg daily on a 3 weeks on, 1 week off schedule)/ fulvestrant $(500 \mathrm{mg}$ administered intramuscularly on day 1 of a 28 -day cycle, with an additional dose on day 15 of the first cycle). The other 242 were randomized to fulvestrant alone. The primary endpoint of median PFS was significantly longer at 20.5 months in the ribociclib/fulvestrant group compared with 12.8 months in the fulvestrant group (HR, 0.59; 95\% CI =0.48-0.732). The treatment effects of ribociclib were similar regardless of whether patients were treatment naive (HR, 0.577) or had one prior line of therapy (HR, 0.565; Slamon et al., 2018). In February 2020, a protocol-specified second interim analysis of MONALEESA-3 reported a significant OS benefit at 42 months with ribociclib/fulvestrant compared with fulvestrant alone ( $57.8 \%$ vs. $45.9 \%$; HR, 0.72; 95\% CI = 0.57-0.92; Slamon et al., 2020).

\section{Abemaciclib}

The single-agent activity of abemaciclib in refractory HR+/HER2 - metastatic breast cancer was established in the phase II, single-arm, MONARCH 1 trial. Of the 132 patients evaluated, the primary endpoint of objective response rate (ORR) was $19.7 \%$. Additionally, $42.4 \%$ of patients had a clinical benefit for $\geq 6$ months. The median PFS and OS rates were 6 months and 17.7 months, respectively (Dickler et al., 2017).

Abemaciclib was also studied in combination with fulvestrant in patients with $\mathrm{HR}+/$ HER2metastatic breast cancer after progression on or within 12 months of adjuvant endocrine therapy or on initial endocrine therapy for metastatic disease in the MONARCH 2 phase III trial, which evaluated the primary endpoint of PFS. Patients were enrolled regardless of menopausal status $(n=669)$ and randomized in a 2:1 fashion to receive either abemaciclib plus fulvestrant vs. fulvestrant alone. Initially, patients were started on abemaciclib $200 \mathrm{mg}$ twice daily; however, safety data indicated the need for a protocol amendment to lower the initial/maximum dose of abemaciclib to 150 mg twice daily. With a median follow-up of 19.5 months, the addition of abemaciclib to fulvestrant resulted in a significantly longer PFS than fulvestrant alone (16.4 months vs. 9.3 months; HR, 0.553; 95\% CI $=0.449-0.681)$. Additionally, the ORR was $48.1 \%$ for patients receiving the abemaciclib/ 
fulvestrant combination vs. $21.3 \%$ for patients receiving fulvestrant/placebo (Sledge et al., 2017). At the time of the initial reporting of the MONARCH 2 trial, OS data had not matured. In June 2019, at the preplanned cutoff for interim OS analysis, the combination of abemaciclib/fulvestrant demonstrated a significant improvement in median OS compared with fulvestrant/placebo (46.7 months vs. 37.3 months, respectively; HR, 0.757; 95\% CI $=0.606-0.945)$. Additionally, the OS benefit was consistent regardless of menopause status or primary vs. secondary endocrine resistance (Sledge et al., 2020).

Lastly, abemaciclib was evaluated in combination with an AI as initial therapy for metastatic breast cancer in postmenopausal women in the phase III MONARCH 3 trial. The primary endpoint was PFS. Patients $(n=493)$ were randomized 2:1 to receive abemaciclib plus an AI (anastrozole or letrozole) or an AI alone. With a median followup of 17.8 months, the addition of abemaciclib to an AI significantly prolonged PFS compared with AI alone (not reached vs. 14.7 months; HR, 0.54; 95\% CI $=0.41-0.72$ ). Additionally, the PFS benefit was consistent in all prespecified subgroups (e.g., metastatic site, receipt of prior endocrine therapy). Of note, OS was not evaluated in MONARCH 3 (Goetz et al., 2017).

\section{ADVERSE EFFECTS}

In the three phase III trials with ribociclib, the most common adverse events were neutropenia $(70 \%-77 \%)$, nausea $(32 \%-52 \%)$, infection $(45 \%-$ $52 \%)$, fatigue $(32 \%-37 \%)$, diarrhea (35\%), and alopecia $(18 \%-33 \%)$. The most common severe adverse events (grade $\geq 3$ ) associated with ribociclib were neutropenia $(53 \%-60 \%)$, increased alanine aminotransferase (ALT; 5\%-9\%), and increased aspartate aminotransferase (AST; 5\%-6\%). Febrile neutropenia was observed in only $1 \%$ to $2 \%$ of patients receiving ribociclib. The most common types of infection observed were of the upper respiratory tract (11\%) and urinary tract (9\%), and limited to grade 1 to $2(39 \%-43 \%)$. Additionally, QTc prolongation $>480$ and $>500$ msec was observed in $3 \%$ to $7 \%$ and $1 \%$ to $2 \%$ of patients, respectively. Dose interruptions and reductions occurred in $77 \%$ and $38 \%$ of patients receiving ribociclib, respectively. Treatment discontinuation due to adverse events occurred in $4 \%$ of patients (Im et al., 2019).

In the two phase III trials with abemaciclib, the most common adverse events observed were diarrhea $(81 \%-86 \%)$, neutropenia $(41 \%-46 \%)$, fatigue (40\%), infection, nausea $(39 \%-45 \%)$, vomiting $(26 \%-28 \%)$, abdominal pain $(29 \%-$ $35 \%)$, anemia $(28 \%-29 \%)$, and loss of appetite $(25 \%-27 \%)$, the majority of which were grade 1 to 2 . The most common severe adverse events (grade $\geq 3$ ) associated with abemaciclib were neutropenia $(21 \%-27 \%)$ and diarrhea (10\%-13\%). The median onset of diarrhea was 8 days, and the median duration was 10.5 days for grade 2 and 8 days for grade 3 . Of note, only $24 \%$ to $30 \%$ of patients required dose modifications, and $2 \%$ to $3 \%$ required treatment discontinuation due to diarrhea. For the majority of patients, neutropenia occurred during the first two cycles, and neutrophil counts remained stable thereafter (Goetz et al., 2017; Sledge et al., 2017).

\section{ROLE IN THERAPY FOR BREAST CANCER}

With FDA-approved indications in both the firstline and second-line settings, treatment with CDK4/6 inhibitors (ribociclib and abemaciclib) provides an alternative approach to cytotoxic therapy for patients with $\mathrm{HR}+/ \mathrm{HER} 2-$ metastatic breast cancer (National Comprehensive Cancer Network, 2020). The OS benefit demonstrated in the MONALEESA-7 and MONARCH 3 trials in the front-line setting validates the importance of using ribociclib and abemaciclib earlier in the treatment course (Goetz et al., 2017; Im et al., 2019). Additionally, PFS rates and duration of response are longer compared with those seen with endocrine therapy.

Ribociclib and abemaciclib are both approved in combination with an AI or fulvestrant plus a gonadotropin-releasing hormone receptor $(\mathrm{GnRH})$ agonist if premenopausal; however, only abemaciclib has approval as single-agent therapy (see Figure 1 for role in therapy). To date, there are no head-to-head trials comparing the efficacy of ribociclib to abemaciclib, and both are considered standard of care for HR+/HER2- metastatic breast cancer (National Comprehensive Cancer Network, 2020). Additionally, both ribociclib and 


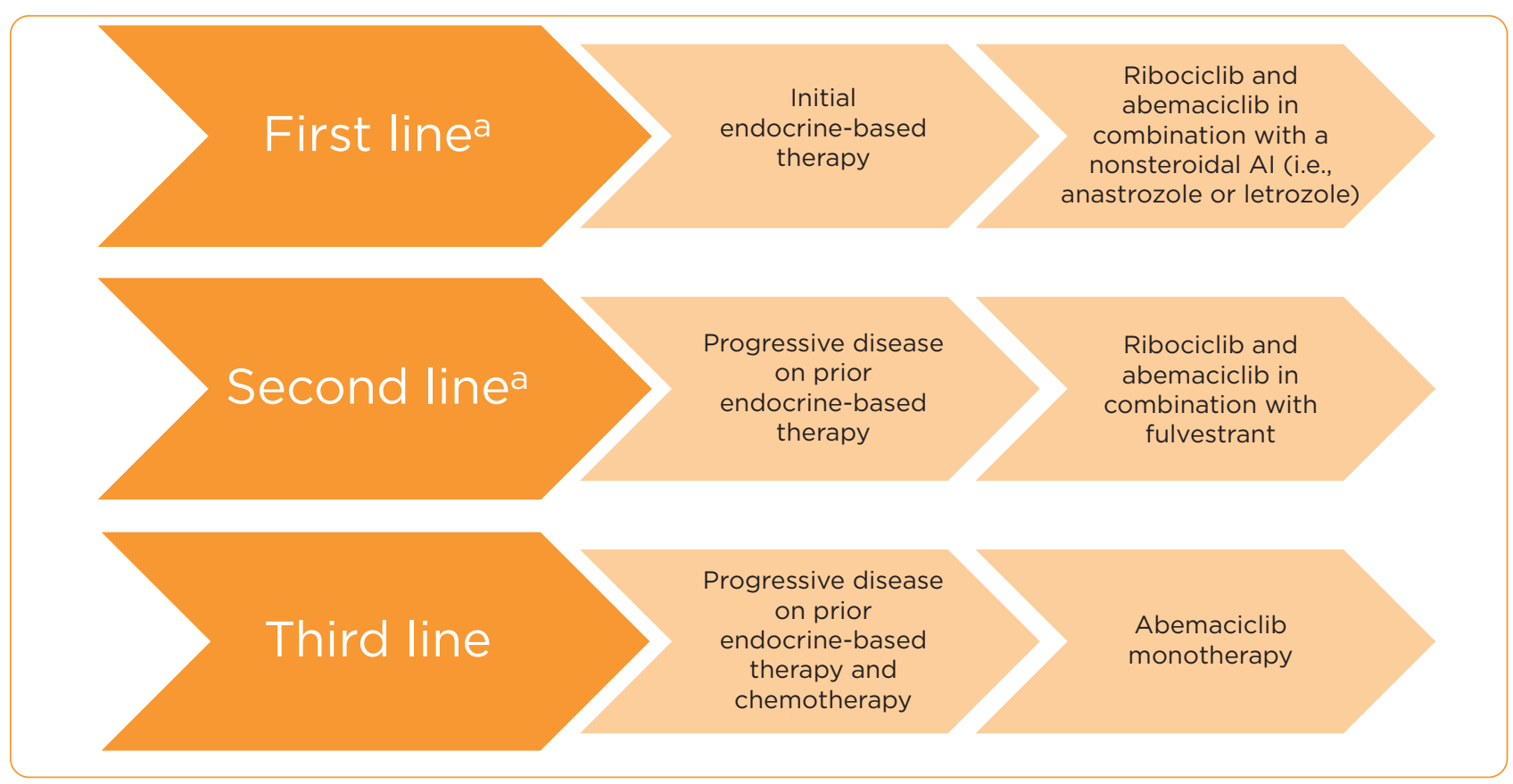

Figure 1. Role of ribociclib and abemaciclib in HR+/HER2- metastatic breast cancer.

$\mathrm{Al}=$ aromatase inhibitor.

${ }^{a}$ Gonadotropin-releasing hormone agonist required if premenopausal.

abemaciclib appear to be well tolerated, with the majority of adverse events being low grade.

\section{IMPLICATIONS FOR THE}

\section{ADVANCED PRACTITIONER}

The incorporation of CDK4/6 inhibitors into the treatment landscape of HR+/HER2- breast cancer provides a convenient oral treatment option that is both effective and well tolerated. Due to growing concern about endocrine resistance and the widespread use of ribociclib and abemaciclib as initial therapy, it is imperative that the oncology advanced practitioner be aware of the benefits and risks of these agents.

The recommended starting dose of ribociclib is $600 \mathrm{mg}$ once daily for 21 days, followed by a 7-day rest period. Ribociclib can be taken without regard to meals but is preferably administered in the morning. Being a major CYP3A4 substrate and a moderate CYP3A4 inhibitor, the potential for drugdrug interactions is substantial. If coadministration with a strong CYP3A4 inhibitor cannot be avoided, ribociclib should be dose reduced to $400 \mathrm{mg}$ daily. Patients should be advised to avoid consumption of grapefruit (juice) while taking ribociclib due to concerns for increased toxicity. Due to the po- tential for QTc prolongation, all patients should undergo ECG monitoring before starting and at the beginning of the first 6 cycles of treatment (see Table 1 for dosing modifications). No dose adjustment is required for estimated glomerular filtration rate (eGFR) $\geq 30 \mathrm{~mL} /$ minute; however, the initial dose should be reduced to $200 \mathrm{mg}$ daily for eGFR $<30 \mathrm{~mL} /$ minute. Additionally, patients with ChildPugh class B or C hepatic dysfunction should receive an initial dose of $400 \mathrm{mg}$ daily.

Overall, ribociclib is well tolerated, and the most common adverse events seen in clinical trials were neutropenia, diarrhea, fatigue, nausea/ vomiting, and infection. Additional laboratory abnormalities commonly seen were AST/ALT and serum creatinine ( $\mathrm{SCr}$ ) elevations. Due to the potential for neutropenia early in the treatment course, the prescribing information recommends monitoring complete blood count (CBC) with differential at baseline, every 2 weeks for the first two cycles, and monthly for the next 2 months at least thereafter (Novartis Pharmaceuticals Corporation, 2020).

The recommended starting dose of abemaciclib is $150 \mathrm{mg}$ twice daily (in combination with an AI) or $200 \mathrm{mg}$ twice daily (as a single agent). 


\section{Table 1. Dose Modifications and Monitoring Parameters of Ribociclib and Abemaciclib}

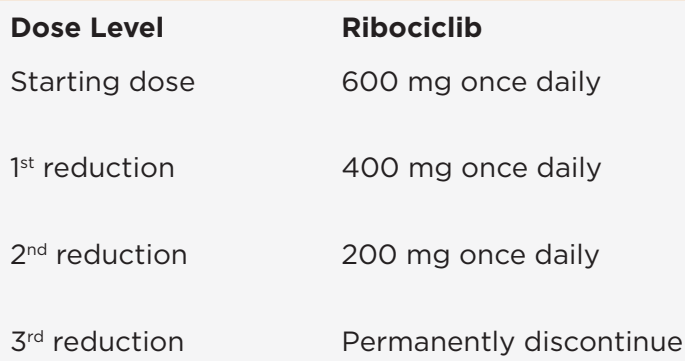

\section{Ribociclib}

No adjustment necessary
Toxicity
Interrupt treatment until recovery to $\leq$ grade 2, then resume at same dose

Interrupt treatment until recovery to $\leq$ grade 2 , then resume at the next lower dose

Interrupt treatment until recovery to $<$ grade 2 , then resume at the next lower dose
Abemaciclib

$150 \mathrm{mg}$ twice daily (with ET)

200 mg twice daily (monotherapy)

$100 \mathrm{mg}$ twice daily (with ET)

$150 \mathrm{mg}$ twice daily (monotherapy)

50 mg twice daily (with ET)

100 mg twice daily (monotherapy)

Permanently discontinue (with ET) $50 \mathrm{mg}$ twice daily (monotherapy)

\section{Abemaciclib}

Neutropenia

Grade 3

Grade 3 recurrent or neutropenic fever

Interstitial lung disease/pneumonitis

Grade 1

No interruption or adjustment necessary.

(asymptomatic)

Initiate appropriate medical management and monitoring as clinically indicated.

Grade 2

(symptomatic)

Interrupt until recovery to $\leq$ grade 1 and consider resuming at next lower dose (if benefit of resuming outweighs risk).

If resolution to baseline or grade 1 occurs within 7 days, no interruption or adjustment necessary.

If recurs, permanently discontinue treatment. If persistent or recurrent, interrupt treatment until toxicity resolves to baseline or grade 1 and resume at next lower dose.
Grade 3 (severe symptomatic) or grade 4 (lifethreatening)

\section{QT prolongation (only applicable to abemaciclib)}

QTCF > $480 \mathrm{msec}$

Interrupt treatment until QTcF resolves to $<481 \mathrm{msec}$, then resume at the next lower dose. If recurs, interrupt treatment until QTcF resolves to $<481 \mathrm{msec}$, then resume at next lower dose.

QTcF > 500 msec

Permanently discontinue treatment.
Interrupt treatment until QTCF resolves to $<481 \mathrm{msec}$, then resume at next lower dose.

If QTCF > 500 msec OR > 60 msec increase from baseline AND associated with torsades de pointes, PVT, unexplained syncope, or signs/symptoms of serious arrhythmia, permanently discontinue treatment.

Diarrhea (only applicable to abemaciclib): At the first sign of loose stools, begin management with antidiarrheal agents and increase oral fluid intake.

Grade 1

Grade 2

Grade 3/4 or requires hospitalization

Note. $\mathrm{ET}$ = endocrine therapy; PVT = polymorphic ventricular tachycardia; $\mathrm{CBC}=$ complete blood count;

LFTs = liver function tests; ECG = electrocardiogram.

No interruption or adjustment necessary.

If toxicity doesn't resolve to $\leq$ grade 1 within 24 hours, interrupt treatment until resolution, then resume at same dose.

If recurs after resumption at same dose (despite maximal supportive care), interrupt treatment until $\leq$ grade 1 , then resume at next lower dose.

Interrupt treatment until $\leq$ grade 1 , then resume at next lower dose. 


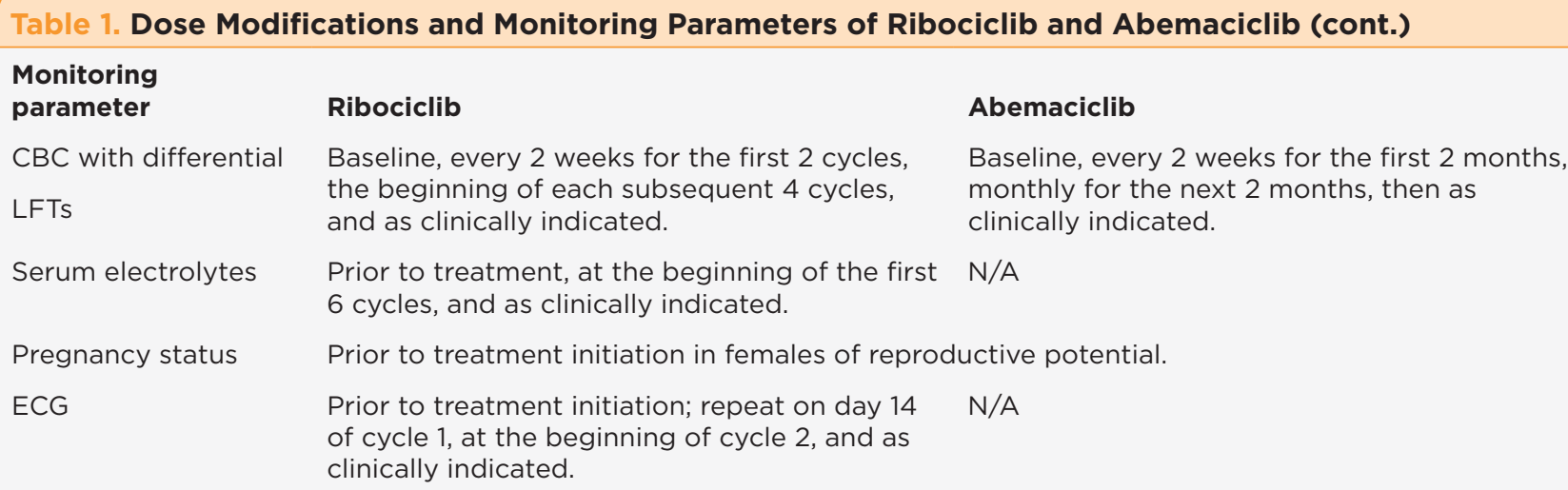

Baseline, every 2 weeks for the first 2 months, monthly for the next 2 months, then as clinically indicated.

Serum electrolytes Prior to treatment, at the beginning of the first N/A 6 cycles, and as clinically indicated.

Pregnancy status Prior to treatment initiation in females of reproductive potential.

ECG

Prior to treatment initiation; repeat on day $14 \quad$ N/A of cycle 1 , at the beginning of cycle 2 , and as clinically indicated.

Note. $\mathrm{ET}=$ endocrine therapy; $\mathrm{PVT}=$ polymorphic ventricular tachycardia; $\mathrm{CBC}=$ complete blood count;

LFTs = liver function tests; ECG = electrocardiogram.

Abemaciclib is also a major CYP3A4 substrate, and concomitant use with the strong inhibitor, ketoconazole, should be avoided. Concomitant use with other strong inhibitors requires dose reductions at $50 \mathrm{mg}$ increments. No dose adjustments are recommended for renal impairment; however, abemaciclib has not been studied in eGFR $<30$ $\mathrm{mL} / \mathrm{minute}$. Additionally, patients with ChildPugh class $\mathrm{C}$ hepatic dysfunction should receive abemaciclib once daily.

Overall, abemaciclib is well tolerated and the most common adverse effects seen in clinical trials were diarrhea, neutropenia, infection, fatigue, nausea/vomiting, and abdominal pain. Similarly to ribociclib, AST/ALT and SCr elevations are common but not typically associated with clinical manifestations. Abemaciclib packaging information recommends monitoring CBC with differential every 2 weeks for the first two cycles and monthly thereafter (see Table 1 for dosing modifications). Because grade 3 diarrhea was common in the first cycle and associated with dehydration and infection, patients should be started on an antidiarrheal agent at the first sign of toxicity and monitored closely (Eli Lilly and Company, 2020).

\section{CONCLUSION}

Ribociclib and abemaciclib are the newest CDK4/6 inhibitors approved for use in $\mathrm{HR}+$, HER2 - metastatic breast cancer. Data from the MONALEESA and MONARCH trial series demonstrated an improvement in both PFS and OS, establishing these agents as standard front-line treatment for advanced disease (Im et al., 2019; Sledge et al., 2020). Both agents are associated with a durable response, with most patients remaining on treatment for an average of 2 years. The favorable toxicity profile and the ease of oral administration help improve quality of life for patients living with an incurable disease. Further investigation is needed to understand mechanisms of resistance to the CDK pathway and between tumor and specific genetics to optimize treatment outcomes.

\section{Disclosure}

Dr. Byers has participated in advisory boards for Heron Therapeutics, Pfizer, Tesaro, and The Delham Group and has received payment from Wellstat Therapeutics for lectures.

\section{References}

Anderson, W., Chatterjee, N., Ershler, W., \& Brawley, O. (2002). Estrogen receptor breast cancer phenotypes in the Surveillance, Epidemiology, and End Results database. Breast Cancer Research Treatment, 76(1), 27-36. https://doi.org/10.1023/a:1020299707510

Dickler, M. N., Tolaney, S. M., Rugo, H. S., Cortes, J., Dieras, V., Patt, D.,...Baselga, J. (2017). MONARCH 1, a phase II study of abemaciclib, a CDK4 and CDK6 inhibitor, as a single agent, in patients with refractory HR+/HER2metastatic breast cancer. Clinical Cancer Research, 23(17), 5218-5224. https://doi.org/10.1158/1078-0432. CCR-17-0754

D’Souza, A., Spicer, D., \& Lu, J. (2018). Overcoming endocrine resistance in metastatic hormone receptor-positive breast cancer. Journal of Hematology \& Oncology, 11, Article number: 80. https://doi.org/10.1186/s13045-0180620-6

Eli Lilly and Company. (2020). Verzenio (abemaciclib) package insert. Retrieved from http://uspl.lilly.com/verzen- 
io/verzenio.html?s=pi

Goetz, M. P., Toi, M., Campone, M., Sohn, J., Paluch-Shimon, S., Huober, J.,...Di Leo, A. (2017). MONARCH 3: Abemaciclib as initial therapy for advanced breast cancer. Journal of Clinical Oncology, 35(32), 3638-3646. https:// doi.org/10.1200/JCO.2017.75.6155.

Hortobagyi, G. N., Stemmer, S. M., Burris, H. A., Yap, Y. S., Sonke, G. S., Paluch-Shimon, S.,...O'Shaughnessy, J. (2016). Ribociclib as first-line therapy for HR-positive, advanced breast cancer. New England Journal of Medicine, 375, 1738-1748. https://doi.org/10.1056/NEJMoal609709

Im, S. S., Lu, Y. S., Bardia, A., Harbeck, N., Colleoni, M., Franke, F.,...Tripathy, D. (2019). Overall survival with ribociclib plus endocrine therapy in breast cancer. New England Journal of Medicine, 381, 307-316. https://doi. org/10.1056/NEJMoa1903765

National Comprehensive Cancer Network. (2020). NCCN Clinical Practice Guidelines in Oncology: Breast cancer. v6.2020. Retrieved from https://www.nccn.org/professionals/physician_gls/pdf/breast.pdf

Novartis Pharmaceuticals Corporation. (2020). Kisqali (ribociclib) package insert. Retrieved from https://www. pharma.us.novartis.com/sites/www.pharma.us.novartis. com/files/kisqali.pdf

Ribnikar, D., Volovat, S., \& Cardoso, F. (2019). Targeting CDK4/6 pathways and beyond in breast cancer. The Breast, 43, 8-17. https://doi.org/10.1016/j. breast.2018.10.001

Siegel, R., Miller, K., \& Jemal, A. (2020). Cancer statistics, 2020. CA: A Cancer Journal for Clinicians, 70(1), 7-30. https://doi.org/10.3322/caac.21590

Slamon, D. J., Neven, P., Chia, S., Fasching, P. A., De Laurentiis, M., Im, S. A.,...Jerusalem, G. (2018). Phase III randomized study of ribociclib and fulvestrant in hormone receptor-positive, human epidermal growth factor receptor 2-negative advanced breast cancer: MONALEESA-3. Journal of Clinical Oncology, 36(24), 2465-2472. https://doi.org/10.1200/JCO.2018.78.9909

Slamon, D. J., Neven, P., Chia, S., Fasching, P. A., De Laurentiis, M., Im, S.-A.,...Jerusalem, G. (2020). Overall survival with ribociclib plus fulvestrant in advanced breast cancer. New England Journal of Medicine, 382, 514-524. https://doi.org/10.1056/NEJMoa1911149

Sledge, G. W., Toi, M., Neven, P., Sohn, J., Inoue, K., Pivot, X.,... Llombart-Cussac, A. (2017). MONARCH 2: Abemaciclib in combination with fulvestrant in women with $\mathrm{HR}+$ / HER2- advanced breast cancer who had progressed while receiving endocrine therapy. Journal of Clinical Oncology, 35(25), 2875-2884. https://doi.org/10.1200/ jco.2017.73.7585

Sledge, G. W., Toi, M., Nevin, P., Sohn, J., Inoue, K., Pivot, X.,... Llombart-Cussac, A. (2020). The effect of abemaciclib plus fulvestrant on overall survival in hormone receptor-positive, ERBB2-negative breast cancer that progressed on endocrine therapy - MONARCH 2. JAMA Oncology, 6(1), 116-124. https://doi.org/10.1001/jamaoncol.2019.4782

Spring, L., Zangardi, M., Moy, B., \& Bardia, A. (2017). Clinical management of potential toxicities and drug interactions related to cyclin-dependent kinase 4/6 inhibitors in breast cancer: Practical considerations and recommendations. The Oncologist, 22(9), 1039-1048. https:// doi.org/10.1634/theoncologist.2017-0142

Tripathy, D., Im, S., Colleoni, M., Franke, F., Bardia, A., Harbeck, N.,...Lu, Y. (2018). Ribociclib plus endocrine therapy for premenopausal women with hormone-receptor positive, advanced breast cancer (MONALEESA-7): A randomised phase 3 trial. Lancet Oncology, 19(7), 904915. https://doi.org/10.1016/S1470-2045(18)30292-4 